\title{
Leveraging Administrative Data for Policy and Programmatic Interventions on Gender Specific Sustainable Development Goals (SDGs)
}

\author{
Compendium of Good and Promising Practices
}

\author{
UN Women East and Southern Africa Regional Office in Nairobi-Kenya
}

\begin{abstract}
The call for a data revolution expressed in the report of the UN Secretary-General's High-Level Panel of Eminent Persons in the lead-up to the 2030 Agenda for Sustainable Development has generated specific attention on the role of data in driving and monitoring sustainable development. Indeed, the availability of and access to highquality, timely, disaggregated, gender-responsive and reliable data, supplemented with contextual information for its interpretation and use, are fundamental to successful monitoring and reporting of the 2030 Agenda and the Leave No One Behind commitment.

In 2016, UN Women launched its flagship initiative to stimulate sustained production and dissemination of quality gender statistics for monitoring the gender-specific Sustainable Development Goal (SDG) indicators. As part of this initiative, UN Women is vigorously pursuing practical, convenient, cost-effective ways to track these indicators. The Flagship Programme Initiative, Making Every Woman and Girl Count (MEWGC), which UN Women launched in September 2016, has stimulated the search for practical, convenient, cost-effective ways to monitor strategic goals aimed at achieving gender equality and women's empowerment. The initiative is directly linked to the targets set for Sustainable Development Goal (SDG) 5, achieve gender equality and empower women and girls, and other gender-specific SDGs. To assess the direct effect of the SDG commitments on gender equality and women's empowerment, 54 gender-specific indicators need to be regularly tracked globally.

Better production and use of gender statistics for evidence-based localization of the SDGs is UN Women's strategy to address the pressing need for better and more gender statistics. In general, the various UN Women regional teams are expected to adapt and implement the global initiatives between 2016 and 2021 by providing direct technical support to countries in their regions-including in pathfinder and selected non-pathfinder countries(based on demand) and working closely with regional partners to help promote and support country-led plans to localize and monitor the SDGs.

The outcome objectives of the MEWGC project are to strengthen the policy and financial environment to enable gender-responsive national adaptation and effective monitoring of the SDGs; improve production of gender statistics to enable monitoring of national policies and reporting commitments under the SDGs; improve the use of gender statistics by different players to inform advocacy, research, policies and programmes; and generate knowledge on cost-effective ways to provide statistical capacity-building in gender statistics in different contexts. Ensuring the availability, accessibility and use of gender statistics to monitor progress in delivering gender equality and women's empowerment commitments in the SDGs and to inform policymaking, advocacy and accountability in any region requires well-coordinated, responsive, effective data systems.

Traditionally, there are three approaches to data gathering: censuses, sample surveys and administrative data collection. These approaches have evolved with the data revolution, particularly in response to the complex needs and demands of the SDGs. Various factors inform the choice of a specific method, including quality, coverage, timeliness, cost and purpose. This is especially true for administrative data, which agencies and institutions primarily collect for non-statistical reasons - i.e. to provide overviews on registration, transactions and record keeping. For example, administrative records are maintained to regulate the flow of goods and people across borders, to respond to legal requirements of registering events such as births and deaths and to administer benefits such as pensions or obligations such as taxation.

Given their importance in the data production process, this study focused solely on administrative data systems. The UN Women 2018 report, Turning Promises into Action: Gender Equality in the 2030 Agenda for Sustainable Development, uncovered a number of bottlenecks in the production and use of gender statistics, which invariably affect or are affected by data quality and timeliness. This study explored the potential of using administrative data to produce gender statistics for monitoring the SDGs.

As such, UN Women regional Office for East and Southern Africa has documented six good and promising practices that are replicable and are feasible within the framework of leveraging use of administrative data for monitoring and reporting on gender specific Sustainable Development Goals. These are just but a few selected examples of how these governments in Africa have used administrative data and civil and vital statistics to accelerate progress towards realizing the gender specific SDGs.

The compendium defines a good practice within the guideline provided for in the Guide-Documenting good
\end{abstract}


practices on gender equality developed by UN Women in 2015. A good practice is "an intervention, business practice, process or methodology that is responsive to the needs of women and girls, replicable, scalable, and succeeds in achieving gender equality and women's empowerment. The essence of identifying and sharing a good practice is to learn from others and to encourage the application of knowledge and experience to new situations as they relate to GEWE. A good practice need not be viewed as prescriptive; it can be adapted to meet new challenges, becoming better as improvements are discovered."

Keywords: SDGs, Administrative data, research, UN Women, East and Southern Africa, good practices, gender equality.

DOI: $10.7176 / \mathrm{PPAR} / 10-8-03$

Publication date:August $31^{\text {st }} 2020$

\subsection{Methodology}

These good and promising practices have been documented from the study on examining the potential of administrative data to produce gender statistics for monitoring gender-specific SDG indicators and to present best practices in the use of gender statistics generated from administrative data for policy interventions. This compendium presents select country case studies of good and promising practices in use of gender statistics generated from administrative data for policy and programme interventions as they relate to gender specific SDGs.

\subsection{Compendium of Good and Promising Practices \\ 3.1 Good and Promising Practice 1: Kenya}

In March 2009, the Kenya Ministry of Gender, Children and Social Development developed a monitoring and evaluation framework for gender mainstreaming. The objective was to provide a structured process that will enable tracking of progress, ensure informed decision-making in the planning and implementation of interventions, inform prudent use of resources and avoid wasting resources, and ensure that none of the key stakeholders duplicate efforts. Consequently, core performance indicators have been identified for each of the stakeholders, including programme implementers, the parastatal management team, gender commissioners and key development partners. These performance indicators are linked to the gender mainstreaming core priority areas and based on the National Policy on Gender and Development (2000); the National Plan of Action to implement the National Gender Policy (2008-2012); the Ministry of Gender, Children and Social Development draft Strategic Plan (20082012); Vision 2030; the Medium-Term Strategy (2008-2012); and the National Integrated Monitoring and Evaluation Strategy developed in 2004. The framework has two main types of indicators: impact and outcomelevel indicators, which measure progress over the long term; and intermediary results and output indicators, which measure the results of the implementation process in each key priority area.

The data source and frequency of data collection and reporting for each of the indicators have been specified, and administrative data feature prominently. The country has developed an E-National Integrated Monitoring and Evaluation Strategy, under the aegis of the Ministry of Devolution and Planning, to capture and present data in real time and in colour code to provide the implementation status of defined SDG indicators at a glance. Similarly, as the coordinator of the NSS, the Kenya NBS has responsibility for coordinating and collaborating with key data producers, including all MDAs that produce gender data as part of their routine activities to compile the required statistics. In line with this, the Kenya NBS has, in collaboration with the Ministry of Public Service, Youth and Gender Affairs and the National Gender Equality Commission, established the Gender Statistics Technical Committee to review priority indicators and validate gender data generated in all sectors of the economy. The government is training statistical officers, planning officers and gender officers at the national and subnational levels to enhance collection, collation and analysis of gender-sensitive data. Box 3.1 reflects some uses of administrative data in Kenya.

In 2015, Kenya produced a gender data sheet involving data from all sources (surveys, administrative data and censuses) to guide policy formulation and planning. A 2015 report provides information on the status of women across various sectors. In addition, Kenya has been tracking budgetary allocation towards women's empowerment endeavours, including women's enterprises.

In terms of specific administrative data use, in 2009, the score for the women's access to land measure was estimated at 1.0, which is defined as impossible. However, in 2014 after the passage and implementation of the 2010 Constitution and the 2012 Land Act, the measure decreased to 0.5, indicating significantly improved access to land for women. Nevertheless, much more needs to be done in terms of access to non-land property, which remained unchanged at 0.5 . Based on these findings, the government has enacted laws to protect women's inheritance of their father's and/or spouse's properties, including land; these laws override customary laws and considerations.

\subsection{Good and Promising Practice 2: Tanzania}

In line with Tanzania's constitution, its Vision 2025 stipulates equality of men and women, and recognizes gender 
equality and the empowerment of women in all socioeconomic and political relations and cultures as one of the strategies to attain this vision. Key national policy frameworks such as the Strategy for Growth and Reduction of Poverty (MKUKUTA II in Tanzania Mainland and MKUZA II in Zanzibar) have identified gender equality and women's empowerment as a major development concern requiring the use of multisectoral approaches. Tanzania's current five-year development plan (2016/17-2020/21) emphasizes women's economic empowerment as a means of bringing about equality in economic empowerment. To ensure that these frameworks are well implemented, the Tanzania Ministry of Health, Community Development, Gender, Elderly and Children has been charged with coordinating and providing guidance for mainstreaming gender in all development processes.

Administrative data has been used in Tanzania as follows. The goal of increasing gender equality and women's empowerment in Tanzania is partly based on recent institutional (administrative) data that suggest that, despite the remarkable progress the country has made in gender equality and women's empowerment, attainment of gender parity among students transitioning to secondary schools, and the growth of women in senior positions (from 33 percent in 2010/11 to 41 percent in 2014/15), the yield per hectare in agriculture was persistently lower on land worked primarily by women, making them poorer than men. In addition, women marry before the age of 18 and start raising families soon after, limiting their education and future employment options. Patriarchal norms largely fuel this situation.

Interventions such as gender-responsive budgeting and the Southern African Development Community Protocol on Gender and Development, which calls for 50/50 representation on all decision-making organs, are being implemented to ensure gender balance and increase women's ability to contribute to overall national socioeconomic transformation. Other strategies in the five-year NDP include rekindling growth in sectors such as agriculture and manufacturing in which the poor tend to derive their livelihoods and which therefore have a strong potential to reduce poverty. Interventions are also in place to combat the low participation of women in economic activities and increase women's economic empowerment, reduce gender income inequality and end child marriage and early pregnancy by 2021 .

\subsection{Good and Promising Practice 3: Uganda}

In Uganda, information from the education and health management information systems and from the annual statistical abstracts that MDAs and higher local governments produce contain gender statistics for national and subnational planning and decision-making. Annual crime reports by the Uganda police force incorporate gender statistics, which have greatly informed policies and interventions on GBV, among others.

In November 2016, the Ministry of Gender, Labour and Social Development launched the National Policy on the Elimination of Gender-Based Violence in Uganda under the theme "Peace in the Home to Peace in the Nation, Making Education Safe for All". The policy aimed to assist stakeholders in increasing their programmatic efforts to prevent and respond to GBV. The underlying reason for this policy came from annual police crime reports, which identified defilement as the leading sex-related crime in the country, with prevalence increasing since 2011. The ministry has also put in place a national GBV database to monitor trends in GBV.

Another study from the Ministry of Education and Sports in 2012 revealed alarming levels of GBV in primary ( 77.7 per cent) and secondary schools ( 82 per cent), resulting in effects such as psychological trauma, sexually transmitted infections, unintended pregnancies, nightmares, and feelings of shame and guilt among the victims. This was in addition to routine corporal punishment meted out to students in schools (74.3 per cent). Sexual abuse was also found to be prevalent in universities and other tertiary institutions; the perpetrators were mostly people who had power over their victims (e.g. teachers, tutors, administrators).

Use of Administrative Data in Uganda entails Uganda's Ministry of Education and Sports which prepared Guidelines on Reporting, Tracking, Referral and Response on Violence against Children. The Ministry of Gender, Labour and Social Development developed National Parenting Guidelines to create awareness among parents to ensure that every child enjoys positive parenting as a human right as enshrined in the Constitution of the Republic of Uganda and the United Nations Convention on the Rights of the Child.

In 2014, the ministry launched the Uganda Child Help Line, a toll-free service that operates 24 hours a day, 7 days a week. People can call in to seek information, formally report cases of violence against children and be referred to nearby authorities and service providers.

\subsection{Good and Promising Practice 4: Ethiopia}

Article 25 of Ethiopia's Constitution states that all persons are equal before the law. Article 35 elaborates, "Women have equal rights with men in all economic, social and political activities". The latest Ethiopia Women's Policy and Development Package was prepared based on these constitutional articles, and the government approved it in March 2017. The main objective of the policy is to create an enabling environment for women to participate at all levels in economic, social and political activities. To ensure that existing data are fully used for planning and decision-making, CSA has started compiling gender statistics from the administrative data generated by sector ministries such as those of health and education. 
Preparation of guidelines for administrative data management by CSA, in collaboration with some sector ministries and UNICEF, is expected to facilitate administrative data production across the country. Box 3.4 illustrates the use of administrative data to monitor gender-specific indicators in Ethiopia. Generally, increasing and emerging demands for data are expected to increase efforts to produce and make administrative data accessible. For example, CSA, in collaboration with UN Women and other stakeholders, has been working to mine data (data extraction) from various sources over time to compute gender-specific indicators. Close relationships and collaborations with the NSS and international agencies will help improve the production and use of gender statistics from administrative data and raise awareness about existing gender inequalities and the need to protect women's rights.

In Ethiopia, administrative data has been harnessed with respect to implementing strategies to enhance gender equality and women's empowerment. For example, women's representation in leadership and decision-making positions has gradually but progressively increased, with women occupying 38.8 per cent of national parliamentary seats at the federal level, 20.6 per cent of the judiciary and 13.3 per cent of decision-making positions in the executive branch.

Substantial improvements in gender parity have also been achieved in education. In the 2014/15 academic year, total primary net enrolment was 90.9 per cent for girls and 97.5 per cent for boys, up from approximately 84 per cent and 90 per cent, respectively, in 2012/13. Also, the share of women enrolled in technical and vocational education and training increased from 48 per cent in 2011/12 to 52 per cent in 2015/16, and the share of women enrolled in undergraduate institutions rose from 27 per cent in 2010/11 to 34 per cent in 2015/16.

Women accounted for 35.2 per cent of public sector employees in 2015/16. Men held 62.6 per cent of jobs created in micro and small enterprises in 2015/16. For women, the largest proportion (26.2 per cent) of jobs created was in construction; for men, it was in urban agriculture (29.4 per cent).

\subsection{Good and Promising Practice 5: Malawi}

The 2015 Malawi National Gender Policy seeks to address several challenges emerging from implementation of the 2000-2005 National Gender Policy, including persistent unequal power relations between men and women and boys and girls due to strong patriarchal attitudes, increasing cases of GBV, high HIV and AIDS infection rates especially in women and girls, limited male involvement in reproductive health and HIV and AIDS programmes, continued high dropout rates for girls from school, high poverty levels particularly among women, limited participation and representation of women in decision-making processes at all levels, inadequate enforcement of laws and huge disparities in access and control over resources by the majority of women. For example, data compiled from administrative sources show that female representation in Parliament dropped from 43 women in 2009 to 32 in 2014. The outlook in terms of gender issues is to increase women's involvement in decision-making positions through merit-based appointment or affirmative action.

\subsection{Good and Promising Practice 6: Rwanda}

In terms of emerging good practices in the use of administrative data to generate gender statistics, Rwanda has established a gender monitoring office that is responsible for monitoring all national and international gender commitments in the private and public sectors using gender-sensitive indicators computed from administrative and other data sources. After the genocide in Rwanda in 1994, the government recognized women as key players in the country's reconstruction and development. Several policies and programmes were introduced to maximize women's participation in the nation's socioeconomic development. These include the Constitution of the Republic of Rwanda (2003) (as revised on 24 December 2015 in Article 10, 4 to enshrine the promotion of gender equality, family promotion and protection of children's rights among the major priorities of the government of Rwanda); Vision 2020 (1998-1999); the New Civil Code; the Economic Development and Poverty Reduction Strategy (2008-2012); the National Gender Policy (2010); and establishment of the Ministry of Gender and Family Promotion, the Gender Monitoring Office, the National Women's Council and the Forum of Rwandan Women Parliamentarians.

Some of the outcomes of these policies based on administrative data are described in the below case studies. Even though enrolment in tertiary education increased between 2011 and 2016, male enrolment was significantly higher than female enrolment (58 per cent versus 42 per cent), suggesting that young women are missing out on opportunities for higher education. Such gender gaps in enrolment at the tertiary level will ultimately increase gender inequality in employment and incomes. This analysis, which the availability and accessibility of up-to-date institutional data have made possible, has opened up opportunities for educational authorities and other policymakers to increase the inclusiveness of education at all levels for male and female students and to ensure that the expected outcomes are achieved.

In terms of use of administrative data in Rwanda, available institutional data suggest that Rwandan policies and programmes led to women securing 61.3 per cent of seats in the Parliament and 38.5 per cent of seats in the Senate in 2018. Rwanda is the first country in the world to record a majority of women in Parliament. Women in 
leadership positions also increased from 22.0 per cent in $2016 / 17$ to 24.5 per cent in $2017 / 18$, and poverty among women fell from 39.1 per cent in 2014 to 38.1 per cent in 2017. Even though maternal mortality dropped from 476 deaths per 100,000 live births in 2010 to 210 in 2014/15, the level is still considered to be high. An audit of maternal deaths by cause showed that, for three consecutive years (2013 to 2015), postpartum haemorrhage was the leading cause of maternal deaths in Rwanda, leading to calls for health providers to be more accountable.

Primary school enrolment achieved gender parity in 2012, with girls' net enrolment (98 per cent) being slightly higher than that of boys (95 per cent); net secondary school enrolment rose from 27 per cent in 2011 to 30 per cent in 2012 for girls and from 24 per cent to 26 per cent for boys. Data for 2017 reveal that net secondary school enrolment continues to be higher for girls ( 36 per cent) than for boys ( 32 per cent).

Despite these achievements, key constraints still hinder the enhancement of gender equality and women's empowerment. For example, women have limited access to credit and wage employment. Following a study on gender and access to finance, in 2017 the Gender Monitoring Office advocated the need for the country to, among other things, invest in financial capacity-building programmes for women to reinforce their ability to act as informed, educated financial consumers.

\subsection{Conclusion}

The best practices case studies indicate that efforts are being made to enhance administrative data quality by improving data production practices, as Ethiopia's National Guidelines on Administrative Data Management and Tanzania's digitization of the birth registration process demonstrate. Similarly, the study countries have used and continue to use a range of gender statistics produced from various types of administrative data to monitor gender equality and women's empowerment and to design policies and programmes to enhance the status of women. In Kenya, this has led to the enactment of laws to protect women's inheritance of their father's or spouse's property, including land. In Uganda, a national gender policy was developed and launched in 2016 based on consistent and increasing reports of defilement and GBV from crime records and education data. In Tanzania, based on the lower yield per hectare of agricultural land cultivated by women than by men, interventions have been put in place to increase women's agricultural output, combat low participation rates of women in economic activities and reduce their poverty level. In Rwanda, on the basis of data generated from the education management information system, policies and programmes were implemented to increase school enrolment and consequently to increase participation in economic activities and reduce poverty levels in women. Ethiopia has developed a women's policy and development package to create an enabling environment for women to participate in all levels of economic, social and political activities. This policy was the result of data that various sector ministries compiled indicating existing gender inequalities. In Malawi, the continuous decline in and limited representation and participation of women in decision-making processes and gender disparities in many socioeconomic domains led to formulation of the 2015 National Gender Policy. Administrative data systems are therefore critical for monitoring and evaluation of progress made in gender equality and women's empowerment indicators in the study countries; they need to be enhanced to ensure that they are comprehensively and efficiently used.

\subsection{Recommendations}

- Governments in the study countries can support the development and strengthening of administrative data systems that contribute to production of gender-specific SDG indicators through regular allocation of funds needed for the following.

- Acquiring infrastructure and tools for data gathering, documentation, storage, security, processing, analysis, dissemination and archiving; for example, National Statistical Institutes can be supported in removing barriers to the development of good Civil Registration and Vital Statistics systems and other administrative data systems, such as by replacing paper-based data collection methods with electronic data capture.

- Establishing statistical units in all Ministries, Departments and Agencies and ensuring that they employ trained statisticians and specifically and regularly allocate annual budgets for their operations.

- $\quad$ Recruiting personnel with the skills necessary for administrative data collection, data management and analysis.

- Ensuring that staff of administrative data systems are equipped with relevant, up-to-date data recording, processing and analytical tools (e.g. computers, personal digital assistants, computer-assisted personal interviewing, open data kits, data digitization, stable Internet systems and statistical software) to increase efficiency in the collection and compilation of gender statistics from these sources.

- Developing protocols and policies to guide gender-sensitive data production and dissemination.

- Providing regular training of personnel responsible for administrative data production on new methodologies needed to measure pertinent emerging issues.

- Helping other National Statistical Institutes to develop national guidelines for administrative data 
management.

\subsection{References}

Ministry of Devolution and Planning, "Implementation of the Agenda 2030 for Sustainable Development in Kenya", Nairobi, 2017; https://www.un.int/kenya/sites/ www.un.int/files/Kenya/vnr report for kenya.pdf.

United Republic of Tanzania, "National Data Roadmap for Sustainable Development: Lessons and Recommendations from 2016-2018”; http://www.ata4sdgs.org/sites/default/files/services_files/Tanzania_Data_Roadmap_Process_for_Sustainabl e Developent_Report_2016-2018.pdf.

Republic of Uganda, "Review Report on Uganda's Readiness for Implementation of the 2030 Agenda. Theme: Ensuring That No One Is Left Behind", 2016; https://sustainabledevelopment.un.org/content/ documents/10689Uganda\%20Review\%20Report_CDs1.pdf.

National Planning Commission, "The 2017 Voluntary National Reviews on SDGs of Ethiopia: Government Commitments, National Ownership and Performance Trends", Addis Ababa, 2017; https://sustainabledevelopment.un.org/content/ documents/16437Ethiopia.pdf.

Government of Malawi, "The Malawi Growth and Development Strategy III (2017-2022): Building a Productive, Competitive and Resilient Nation", Lilongwe, 2017.

Guidance Note on documenting good practices on Gender Equality and Women's Empowermenthttps://africa.unwomen.org/en/digital-library/publications/2017/07/guidance-note-on-documenting-goodpractices-on-gewe

United Nations, "Rwanda Voluntary National Review, 2019"; https://sustainabledevelopment.un.org/ memberstates/rwanda.

SDG Indicators: Metadata repository (https://unstats. un.org/sdgs/metadata/; accessed 19 December 2018).

Republic of Rwanda. "Rwanda Civil Registration and Vital Statistics Systems. Comprehensive Assessment Final Report, Volume I", 2016.

Rwanda Law No. 32/2016 of 28/08/2016; Zanzibar Births and Deaths Registration Act, No.10 of 2006; Malawi National Registration Act, 2009; Tanzania Births and Deaths Registration Act, 2002; Federal Republic of Ethiopia. Registration of Vital Event and National Identity Card, Proclamation No. 760/2012; Kenya Births and Deaths Registration Act of 1928.

CIVICUS, "Citizen-Generated Data for Sustainable Development Goal: Lessons Learnt from SDG5 Monitoring in Kenya and Tanzania", 2016; http://civicus.org/thedatashift/wp-content/ uploads/2016/10/BriefingonCountryLevelMonitoringo fSDGs.pdf.

United Republic of Tanzania, "Data Quality Assessment Framework for Tanzania Mainland", National Bureau of Statistics, Ministry of Finance, Dar es Salaam, 2014.

National Institute of Statistics of Rwanda, "National Quality Assurance Framework", http://statistics.

Republic of Malawi, "National Gender Policy, Second Edition”, 2015.

UNICEF, "Children’s Rights and Sustainable Development in Rwanda: A Situation Analysis", 2017.

\section{Acknowledgement}

The compendium has been developed for UN Women East and Southern Africa by Jack Onyisi Abebe-Regional Knowledge Management and Research Specialist-UN Women East and Southern Africa Regional Office and Michael Faraday-Regional Knowledge Management and Research Assistant-UN Women East and Southern Africa Regional Office.

The compendium has emerged from a UN Women study on Advancing Administrative Sources of Data for Monitoring Gender-Specific Sustainable Development Goals in Africa led by Philomena Efua Nyarko and Fatmata Lovetta Sesay. It is meant to enhance peer learning from amongst African countries on what works in diverse jurisdictions regarding administrative data.

View the report at: http://africa.unwomen.org/en/digital-library/publications

The views expressed in this publication are those of the authors and do not necessarily represent the views of UN Women, the United Nations or any of its affiliated organizations.

(C) UN Women 2020. All rights reserved.

The views expressed in this compendium are those of the author(s) and do not necessarily represent the views of UN Women, the United Nations or any of its affiliated organizations. 